\title{
Article \\ Machine and Deep Learning Applied to Predict Metabolic Syndrome without a Blood Screening
}

\author{
Guadalupe O. Gutiérrez-Esparza ${ }^{1,2}{ }^{\oplus}$, Tania A. Ramírez-delReal ${ }^{1,3}{ }^{\oplus}$, Mireya Martínez-García ${ }^{2}$, \\ Oscar Infante Vázquez ${ }^{2}$, Maite Vallejo ${ }^{2, *}$ [D and José Hernández-Torruco ${ }^{4, *}$ (D) \\ 1 Cátedras CONACYT Consejo Nacional de Ciencia y Tecnología, Ciudad de Mexico 08400, Mexico; \\ ggutierreze@conacyt.mx (G.O.G.-E.); tramirez@centrogeo.edu.mx (T.A.R.-d.) \\ 2 Instituto Nacional de Cardiología Ignacio Chávez, Ciudad de México 14080, Mexico; \\ mireya.martinez@cardiologia.org.mx (M.M.G.); osinfa@yahoo.com (O.I.V.) \\ 3 Centro de Investigación en Ciencias de Información Geoespacial, Circuito Tecnopolo Norte No. 107, \\ Col. Tecnopolo Pocitos II, Aguascalientes 20313, Mexico \\ 4 División Académica de Ciencias y Tecnologías de la Información, Universidad Juárez Autónoma de Tabasco, \\ Cunduacán, Tabasco 86690, Mexico \\ * Correspondence: maite.vallejo@cardiologia.org.mx (M.V.); jose.hernandezt@ujat.mx (J.H.-T.)
}

Citation: Gutiérrez-Esparza, G.O.; Ramírez-delReal, T.A.; Martínez-García, M.; Infante Vázquez, O.; Vallejo, M.; Hernández-Torruco, J. Machine and Deep Learning Applied to Predict Metabolic Syndrome without a Blood Screening. Appl. Sci. 2021, 11, 4334. https://doi.org/10.3390/app11104334

Academic Editor: Federico Divina

Received: 1 April 2021

Accepted: 6 May 2021

Published: 11 May 2021

Publisher's Note: MDPI stays neutral with regard to jurisdictional claims in published maps and institutional affiliations.

Copyright: (c) 2021 by the authors. Licensee MDPI, Basel, Switzerland. This article is an open access article distributed under the terms and conditions of the Creative Commons Attribution (CC BY) license (https:/ / creativecommons.org/licenses/by/ $4.0 /)$.

\begin{abstract}
The exponential increase of metabolic syndrome and its association with the risk impact of morbidity and mortality has propitiated the development of tools to diagnose this syndrome early. This work presents a model that is based on prognostic variables to classify Mexicans with metabolic syndrome without blood screening applying machine and deep learning. The data that were used in this study contain health parameters related to anthropometric measurements, dietary information, smoking habit, alcohol consumption, quality of sleep, and physical activity from 2289 participants of the Mexico City Tlalpan 2020 cohort. We use accuracy, balanced accuracy, positive predictive value, and negative predictive value criteria to evaluate the performance and validate different models. The models were separated by gender due to the shared features and different habits. Finally, the highest performance model in women found that the most relevant features were: waist circumference, age, body mass index, waist to height ratio, height, sleepy manner that is associated with snoring, dietary habits related with coffee, cola soda, whole milk, and Oaxaca cheese and diastolic and systolic blood pressure. Men's features were similar to women's; the variations were in dietary habits, especially in relation to coffee, cola soda, flavored sweetened water, and corn tortilla consumption. The positive predictive value obtained was $84.7 \%$ for women and $92.29 \%$ for men. With these models, we offer a tool that supports Mexicans to prevent metabolic syndrome by gender; it also lays the foundation for monitoring the patient and recommending change habits.
\end{abstract}

Keywords: metabolic syndrome; features selection; health parameters; random forest; deep neural networks; C4.5; Mexico City; tlalpan 2020 cohort

\section{Introduction}

Nowadays, chronic degenerative diseases, such as ischemic heart disease, type 2 diabetes mellitus, and cerebrovascular stroke, are the leading causes of morbidity and mortality worldwide; these diseases share one or more metabolic components (glucose intolerance, insulin resistance, central obesity, dyslipidemia, and hypertension) that might co-exist in one individual. The term Metabolic Syndrome (MetS) was coined to express this constellation of metabolic abnormalities [1]. The prevalence of MetS in Mexico is 41\% [2] higher than in developed countries, like the United States (34.2\%) [3], due to the epidemic proportion that overweight and obesity have taken in our country, affecting not only the adult population, but also young individuals and even children, with obesity being a central, key component of MetS.

The evaluation criteria for MetS have been proposed by the National Cholesterol Education Program Adult Treatment Panel III (NCEP ATP III) [4], the International Diabetes 
Federation (IDF) [5,6], and the World Health Organization (WHO) [7]. In order to establish a clinical diagnose of MetS, at least three metabolic abnormalities mostly co-occurr in the same individual: elevated blood sugar levels, insulin resistance, abdominal obesity, high blood pressure, and abnormal lipid profile (high blood levels of triglyceride and low blood levels of high-density lipoprotein cholesterol).

These evaluation criteria have been useful from a clinical point of view. However, from the general population perspective, its utility is limited, since laboratory screening is needed [8], which can be costly or cumbersome to implement for certain population levels. The above and the fact that most people are unaware of their health condition, and approach health services until the sickness has caused health limitations [9-11]. For this reason, it is essential to generate tools that can help people to identify pre-clinical conditions, so that preventive measures can be taken at a population level.

The application of machine learning and deep learning (DL) algorithms has facilitated the construction of effective models to predict disease diagnoses and the corresponding treatments $[12,13]$. However, the appropriate selection of variables (potential risk factors) is decisive in improving the models [14-16].

In the case of MetS, the use of machine learning and deep learning algorithms, as well as feature selection methods, have achieved significant results in the development of models to support the prediction of this syndrome $[17,18]$. Related studies that have omitted blood screening for the diagnosis of the MetS have had promising results. An investigation conducted by Barrios et al. [19], proposed a data mining methodology to diagnose MetS without blood screening by applying Artificial Neural Networks (ANN).

In that work, hip circumference, dichotomous waist circumference, dichotomous blood pressure, and sex were included; the specificity reported was $82.59 \%$, a Positive Predictive Value (PPV) of $90.54 \%$, and an Area under the Receiver Operating Characteristic (ROC) Curve of $87.36 \%$. Romero et al. [20] constructed a model applying ANN and considering variables, such as Body Mass Index (BMI), Waist Circumference (WC), weight, height, and sex. The authors used PPV as an evaluation metric, obtaining a value of $38.8 \%$.

Ivanović et al. [21] presented a model using ANN for the prediction of MetS, excluding blood screening. The features that result in this study were: gender, age, BMI, Waist-toheight Ratio (WhtR), systolic, and diastolic blood pressures, with a PPV of $85.79 \%$ and Negative Predictive Value (NPV) of $83.19 \%$.

Another study relates the dietary habits with MetS in the Swedish region. The authors used chi-square analysis [22]. They found four patterns that were defined by clusters that demonstrated a strong association between food and components of MetS. For example, hyperglycemia in men was associated with cheese, cake, and alcoholic beverages consumption; a higher risk of hyperinsulinemia and dyslipidemia in women was associated with white bread consumption. That work was relevant, since different patterns were used for each gender.

The purpose of this article is to identify the most relevant features to propose a risk predictor for the early detection of people with MetS, through machine learning algorithms, such as Random Forest (RF), Ripper (C4.5), and deep neural networks, when considering traditional risk factors for this syndrome as well as dietary information [23,24] and habits, like the consumption of alcoholic beverages [25], smoking [26], physical activity [27], and quality of sleep [28].

This paper is structured, as follows: in Section 2, we introduce the materials and methods. In Section 3, we present the experiments performed and the results. Section 4 shows the discussion, and, finally, the conclusions.

\section{Materials and Methods}

\subsection{Data}

The data set used in this research was collected from the Tlalpan 2020 cohort, a study that was conducted by the Instituto Nacional de Cardiología Ignacio Chávez in Mexico City [29]. The Tlalpan 2020 cohort was approved by the Institutional Bioethics Committee 
of the Instituto Nacional de Cardiología-Ignacio Chavez (INC-ICh) under code 13-802. This work consists of 2289 subjects between 20 and 50 years old, 1369 women, and 920 men, it is important to mention that informed consent was obtained from all the participants. The prevalence of MetS, according to NCEP ATP III criteria, was $24.4 \%$ higher in women (54\%) than in men $(46 \%)$. This data set includes health parameters and habits that are related to alcohol consumption, smoking, physical activity, dietary, and quality of sleep.

\subsubsection{Clinical and Anthropometric Parameters}

Systolic and diastolic blood pressure were measured according to The Seventh Report of the Joint National Committee on the Prevention, Detection, Evaluation, and Treatment of High Blood Pressure (JNC 7) standard procedure [30]. WC, height, and weight were measured according to The International Society for the Advancement of Kinanthropometry (ISAK) [31], BMI was calculated as weight/height ${ }^{2}$ and $\mathrm{WHtR}$ was calculated as the ratio of waist and height (wast/height) $(\mathrm{cm})$.

\subsubsection{Biochemical Evaluation}

The blood samples were taken after $12 \mathrm{~h}$ of overnight fasting, and the following laboratory tests measurements were obtained: fasting plasma glucose (FPG), triglycerides (TGs), and HDL cholesterol (HDL-C) cholesterol LDL (LDL-C), total cholesterol (T-C).

\subsubsection{Dietary Information}

Dietary information was collected using the nutrient software program known as Sistema de Evaluación de Hábitos Nutricionales y Consumo de Nutrimientos (SNUT) (Evaluation of Nutritional Habits and Nutrient Consumption System) [32], as developed by the Instituto Nacional de Salud Pública de México (National Institute of Public Health of Mexico). The SNUT includes data from various types of nutrients classified into different categories, such as dairy products, fruits, meats, vegetables, legumes, cereals, sweets and candies, beverages, fats, cravings, and others. All of the variables of the SNUT were included, and their nominal numerical value was considered, which is, the frequency of food consumption during the day, in the last year.

\subsubsection{Habits}

The following lifestyle information was collected; furthermore, they are inputs for the machine and deep learning algorithms: (1) the smoking habit that was summed up as never smoked, former, or current smoker, and the three features are dichotomous. (2) Alcohol consumption that was classified as a current drinker (dichotomous variable), frequency alcohol consumption, and cups or beers consumed on average when drinking alcohol; these last two are considered to be numeric variables according to the weekly ingesting. (3) Physical activity was obtained by the extended version of the International Physical Activity Questionnaire, IPAQ, [33] that measures the level of physical activity as low, moderate, and high, through questions regarding four domains: work, home, transportation, and leisure time; for this paper, we used the nominal numerical value of variables that are related with leisure time domain, like the days and duration of the type of physical activity (walking, moderate, or vigorous) per week in the last seven days. Lastly (4), quality of sleep was obtained employing the Medical Outcomes Study-Sleep Scale (MOSS) [34], we consider the nominal numerical value of these variables, where the values are described in [35].

\subsection{Methods}

For the study, we applied three machine learning algorithms, RF, C4.5, and deep learning. We conducted experiments for each sex. We choose these machine learning algorithms because of their excellent results in many applications and because each one uses a different approach in a classification task $[18,19,36]$. We also performed feature selection to search 
for simpler and better models. The experiments were conducted using $\mathrm{R}$ programming language [37]. We aimed to predict the MetS without using biochemical variables.

Initially, the parameters of the biochemical evaluation, as well as the anthropometric factors: waist circumference, systolic and diastolic blood pressure, glucose, high-density lipoprotein cholesterol (HDL), and triglycerides, in order to identify and classify participants with MetS based on the definition that was established by the NCEP ATP III. Once the participants have been classified as positive and negative concerning the MetS, the input dataset for the learning algorithms is generated, being composed of categories related to clinical and anthropometric parameters and habits, like alcohol consumption, smoking, physical activity, dietary, and quality of sleep.

The performance of the models was evaluated according to accuracy, balanced accuracy, PPV, and NPV. Figure 1 shows a block diagram of the prediction model and describes the general methodology applied. As a first step, the instances of the data set were classified using the biochemical evaluation data and the clinical and anthropometric parameters, according to the NCEP ATP III criteria. Subsequently, we separated men and women to obtain the most important variables for each sex.

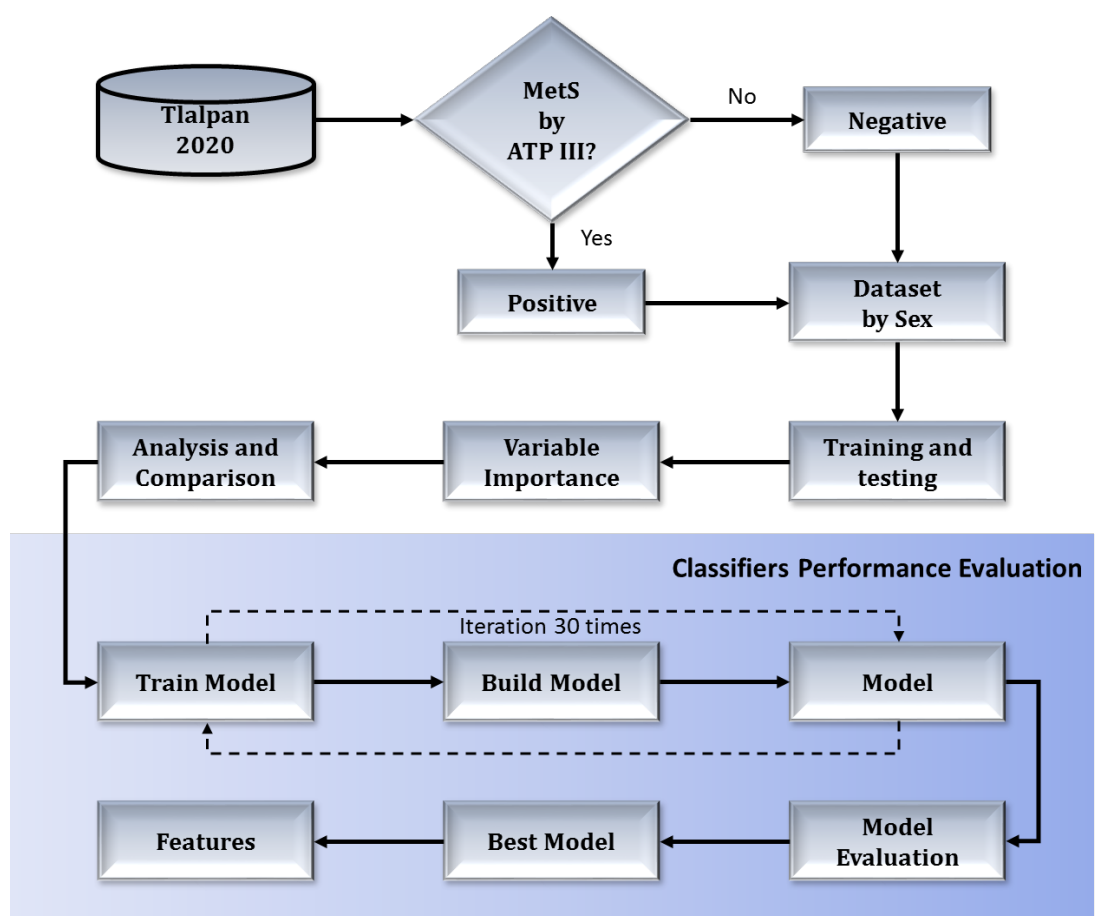

Figure 1. Prediction Model.

We applied RF to identify and evaluate the variable importance by category, as well as Pearson Correlation Coefficient (PCC) and chi-square, to obtain the most important variables when considering all of the categories together.

We created and evaluated predictive models using the ML algorithms for different subgroups that formed based on the variables obtained. From these models, we selected the most relevant features for men and women.

In all experiments, we performed 30 independent runs by algorithm, which is a typical number that is used in the literature for fair comparisons among experiments [38,39]. Subsequently, we calculated the mean and standard deviation for each metric.

\subsubsection{Random Forest}

$\mathrm{RF}$, as introduced by Breiman [40], is a machine learning algorithm that is based on a combination of tree estimators that operate as an ensemble for classification and regression cases. In this work, this method was used for the classification and identification of the 
importance of the variables, based on the mean decrease impurity [41], which is a method that is measured by the Gini index for variable $x_{j}$, and it is computed by the equation:

$$
V I=\left(X_{j}\right)=\frac{1}{n_{\text {tree }}}\left[1-\sum_{k=1}^{n \text { tree }} \operatorname{Gini}(j)^{k}\right]
$$

where $n$ tree is the number of trees.

\subsubsection{C4.5}

C4.5 builds a decision tree from training data using recursive partitions. In each iteration, C4.5 selects the attribute with the highest gain ratio as the attribute from which the tree is branched. This results in a more simplified tree [42,43]. In order to obtain the gain ratio, the following calculations are needed:

$$
\text { Entropy } H(S)=-\sum_{i=1}^{m} p_{i} \log _{2} p_{i}
$$

where $S$ is a set consisting of $s$ data samples with $m$ distinct classes, and $p_{i}$ is the probability that an arbitrary sample belongs to class $C_{i}$.

Let attribute $A$ have $v$ distinct values. The entropy, or expected information based on the partitioning into subsets by $A$, is given by:

$$
\begin{aligned}
& \text { Information Gain } I(S, A)=H(S)-H(S \mid A) \\
& \operatorname{SplitInfo}_{A}(S)=\sum_{i=1}^{v}\left(\left|S_{i}\right| /|S|\right) \log _{2}\left(\left|S_{i}\right| /|S|\right)
\end{aligned}
$$

SplitInfo represents the information that is generated by splitting the training data set $S$ into $v$ partitions, corresponding to $v$ outcomes of a test on the attribute $A$.

Finally, the gain ratio is defined, as follows:

$$
\text { GainRatio = InformationGain/SplitInfo }
$$

\subsubsection{Chi-Squared}

Chi-Squared is a statistical test that is used in machine learning to identify the essential features in a dataset for a classification task [44]. It gives a feature ranking as a result. Taking a feature $f$ and the class $c(\bar{f}, \bar{c}$ as complements), the chi-squared test is computed with the equation:

$$
X^{2}(f, c)=\frac{N[P(f, c) P(\bar{f}, \bar{c})-P(f, \bar{c}) P(\bar{f}, c)]^{2}}{P(f) P(\bar{f}) P(c) P(\bar{c})}
$$

where $N$ is the number of records in the dataset. $P(x, y)$ is the joint probability of $x$ and $y$. $P(x)$ is the marginal probability of $x$.

\subsubsection{Pearson Correlation}

For the statistical analysis, the PCC was used to filter features [45]. Those with a correlation coefficient above 0.5 were used to train machine or deep learning algorithms. This relation is defined by Equation (7) [46]:

$$
p c c\left(u, u^{\prime}\right)=\frac{\sum_{i \in I}\left(r_{u, i}-\bar{r}_{u}\right)\left(r_{u^{\prime}, i}-\bar{r}_{u^{\prime}}\right)}{\sqrt{\sum_{i \in I}\left(r_{u, i}-\bar{r}_{u}\right)^{2}} \sqrt{\sum_{i \in I}\left(r_{u, i}-\bar{r}_{u^{\prime}}\right)^{2}}},
$$

where $r_{u, i}$ and $r_{u^{\prime}, i}$ are the rating scores, also $\bar{r}_{u}$ and $\bar{r}_{u^{\prime}}$ are the average ratings. In this work, we used PCC to find the highest correlation between the health parameters and, thus, select the most relevant features, as we mentioned before, with a threshold of 0.5 . Additionally, it is essential to highlight and the output corresponds to the classification in 
the dataset labeled by NCEP ATP III criteria. With this procedure, we aimed at improving the performance of the predictive models.

\subsubsection{Deep Neural Networks}

The basis for the development of deep learning is ANN, that through the connection among many hidden layers, can learn features to train the model [47]. In this work, Keras was used [48]; this model is based on a sequential class; it means that the network is created layer by layer. Only the dimension for the first layer corresponds to the number of features; then, the hidden layers' dimension is deeply connected to the neural network. The first layer consists of a convolution network with an input shape of seven (features selected); after the first layer's output is flattened, the purpose is to only have one dimension in the shape output. The third layer is a dense network with a dimension of 8 , another dense network (dimension $=8$ ) is added; finally, the last layer is a dense network; all of them have a sigmoid function for activation. Figure 2 shows the general configuration for the deep neural network implemented. The training parameters were a learning rate of 0.0001 and 2500 epochs with Adam optimization. The parameters were selected according to proof of good performance.

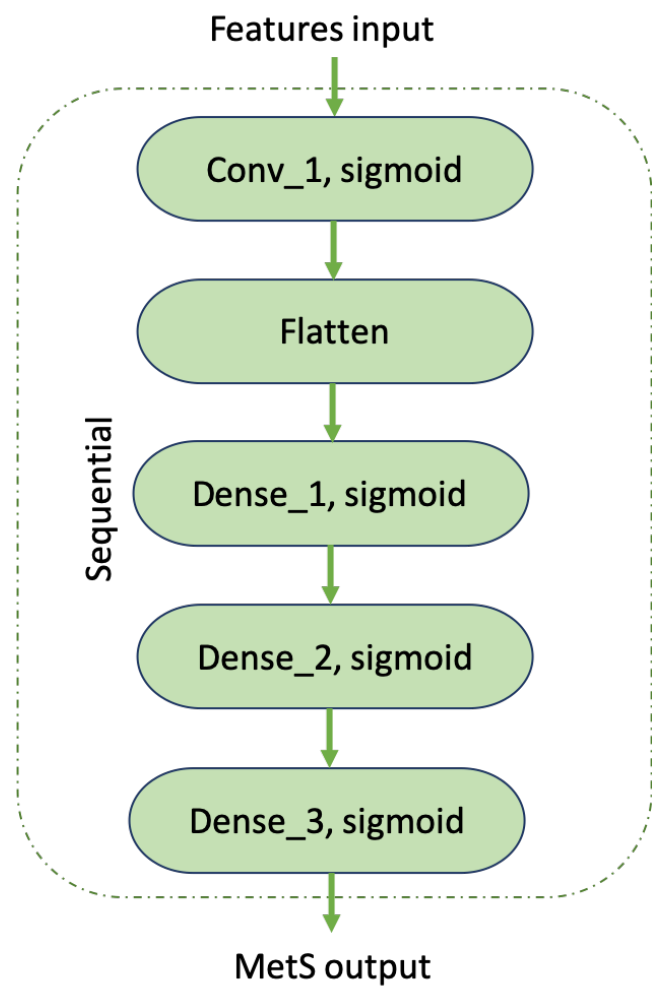

Figure 2. Deep Learning Model.

\subsection{Metrics}

For the evaluation of the model performance, we used the $P P V, N P V$, accuracy $(A C C)$, and balanced accuracy (B.ACC).

$$
\begin{aligned}
P P V & =\frac{T P}{T P+F P} \\
N P V & =\frac{T N}{F P+F N} \\
A C C & =\frac{T P+T N}{P+N}
\end{aligned}
$$




$$
\text { B.ACC }=\left(\frac{1}{2}\right)\left(\frac{T P}{P}+\frac{T N}{N}\right)
$$

where $P=$ Positive, $N=$ Negative,$T P=$ True Positive, $F N=$ False Negative, $T N=$ True Negative, and $F P=$ False Positive, respectively.

\section{Results}

\subsection{Variable Importance by Category}

The first step was to separate the men and women data, then the essential variables by gender and category were obtained:

- clinical and anthropometric parameters,

- dietary information,

- quality of sleep, and

- habits (smoking, alcohol consumption, physical activity).

Applying the variable importance measures (VIM) of RF.

Tables $1 \mathrm{~A}$ and $2 \mathrm{~A}$ show the most important variables (in descending order) obtained for women and men, respectively.

\subsection{Variable Importance of the Complete Dataset}

As second step, the most important variables for women and men were obtained when considering the whole dataset. For this process, VIM of RF, chi.square, and PCC were applied, the resulting variables were compared with those that were obtained for each category to identify which ones were repeated in each case.

Tables $1 \mathrm{~B}$ and 2B show the most important variables (in descending order) for women and men while considering the whole dataset.

\subsection{Most Important Variables in Women}

We found that the five most important variables for women in the feeding habits category were: medium cola soda (cola_soda), soda (flav_soda), Oaxaca cheese (oax_cheese), a cup of coffee (c_coffe), and flavored water (flav_water). In the quality of sleep, the five most important variables were: snoring during sleep (snore), restless sleep (restless), little naps (lit_sleep), not getting enough sleep (nt_sleep), and feeling drowsy (drowsy). The five most important variables for the clinical and anthropometric parameters were: WC, DBP, WHtR, BMI, and SBP. In the category of habits, the most important variable was the cups or beers consumed on average when drinking alcohol (EtOH.avg), followed by smoke (smk.smke), and, finally, the free physical activity (exrcs). Table 1A presents these results.

Now, when considering the whole dataset, we applied VIM of RF, PCC, and chi.square to obtain the most important variables (see Table 1B). In the case of VIM of RF, it identified twelve variables, which were: age, waist, BMI, WHtR, weight, height, SBP, DBP, cola_soda, snore, oax_cheese, and c_coffe. Moreover, the PCC method determined ten variables, which were: age, weight, BMI, waist, WHtR, SBP, DBP, snore, cola_soda, and pork rind (pork_r). Finally, chi.square obtained nine variables: age, BMI, waist, WHtR, weight, SBP, DBP, snore, and cola_soda. Table 1B presents these results. 
Table 1. (A) Most important variables in women by category. (B) Most important variables in women in whole dataset.

\begin{tabular}{|c|c|c|}
\hline Category & Method & Resulting variables \\
\hline \multicolumn{3}{|l|}{ (A) } \\
\hline Feeding habits & VIM of RF & cola_soda, flav_soda, oax_cheese, c_coffe and flav_water \\
\hline Quality of sleep & VIM of RF & snore, restless, lit_sleep, restless and drowsy \\
\hline Anthropometry & VIM of RF & Waist, WHtR, BMI, SBP, DBP, and Age \\
\hline Habits & VIM of RF & EtOH_AVG, smk.smke and exrcs \\
\hline \multicolumn{3}{|l|}{ (B) } \\
\hline \multirow{3}{*}{ Whole Dataset } & VIM of RF & $\begin{array}{l}\text { Age, Waist, BMI, WHtR, Weight, Height, SBP, DBP, } \\
\text { cola_soda, snore, oax_cheese and c_coffe }\end{array}$ \\
\hline & PCC & $\begin{array}{l}\text { Age, Weight, BMI, Waist, WHtR, SBP, DBP, snore, cola_soda } \\
\text { and pork_r }\end{array}$ \\
\hline & Chi.square & $\begin{array}{l}\text { Age, BMI, Waist, WHtR, Weight, SBP, DBP, snore and } \\
\text { cola_soda }\end{array}$ \\
\hline
\end{tabular}

(A) cola_soda: medium cola soda,flav_soda: soda, oax_cheese: Oaxaca cheese, c_coffe: cup of coffee, flav_water: flavor water, snore: snore during sleep, restless: restless sleep, lit_sleep: sleeps little, nt_sleep: not getting enough sleep, drowsy: feeling drowsy, EtOH_AVG: cups or beers consumed on average when drinking alcohol, smk.smke: smoke, exrcs: free physical activity. BMI: Body Mass Index, WHtR: Waist-to-Height-Ratio, SBP: systolic blood pressure, DBP: diastolic blood pressure. (B) cola_soda: medium cola soda, oax_cheese: Oaxaca cheese, c_coffe: cup of coffee, pork_r: pork rind, snore: snore during sleep, BMI: Body Mass Index, WHtR: Waist-to-Height-Ratio, SBP: systolic blood pressure, $D B P$ : diastolic blood pressure.

\subsection{Most Important Variables in Men}

In relation to feeding habits, the five most important variables were: cola_soda, flav_water, hot sauce or chili (hot_chili), c_coffe, and corn tortilla (tortilla). In the case of clinical and anthropometric parameters were: waist, WHtR, BMI SBP, and DBP. In the category of habits, the essential variable was smoke smk.smke, followed by EtOH.avg, and exrcs, finally in the case of quality of sleep, the five most important variables were: snore, nt_sleep, drowsy, restless, and tired during the day (tired). Table 2A shows these results.

Using the whole dataset, we found similar important variables applying VIM of RF, PCC, and Chi.square (see Table 2B). With VIM of RF, the following variables were identified: DBP, waist, BMI, WHtR, SBP, weight, age, snore, cola_soda, flav_water, c_coffe, and tortilla. Applying PCC, the most important variables were: age, weight, BMI, waist, WHtR, SBP, DBP, snore, and cola_soda.

Table 2. (A) Most important variables in men by category. (B) Most important variables in men in whole dataset.

\begin{tabular}{|c|c|c|}
\hline Cathegory & Method & Resulting Variables \\
\hline \multicolumn{3}{|l|}{ (A) } \\
\hline Feeding habits & VIM of RF & cola_soda, flav_water, hot_chili, c_coffe and tortilla \\
\hline Quality of sleep & VIM of RF & snore, nt_sleep, SLP SMN, restless and tired \\
\hline Anthropometry & VIM of RF & DBP, Waist, SBP, WHtR, BMI and Age \\
\hline Habits & VIM of RF & smk.smke, EtOH_AVG and exrcs \\
\hline \multicolumn{3}{|r|}{ ( } \\
\hline \multirow{3}{*}{ Whole Dataset } & VIM of RF & $\begin{array}{l}\text { DBP, Waist, BMI, WHtR, SBP, Weight, Age, snore, } \\
\text { cola_soda, flav_water, c_coffe and tortilla }\end{array}$ \\
\hline & PCC & $\begin{array}{l}\text { Age, Weight, BMI, Waist, WHtR, SBP, DBP, } \\
\text { snore and cola_soda }\end{array}$ \\
\hline & Chi.square & $\begin{array}{l}\text { BMI, Waist, DBP, SBP, Weight, WHtR, Age, } \\
\text { snore and cola_soda }\end{array}$ \\
\hline
\end{tabular}

(A) cola_soda: medium cola soda, flav_water: a glass of flavored sweetened water, hot_chili: a tablespoon of hot sauce or chili in food, c_coffe: a cup of coffee, tortilla: corn tortilla, snore: snore during sleep, nt_sleep: not getting enough sleep, drowsy: feeling drowsy, restless: restless sleep, tired: feel fatigue, smk.smke: smoke, EtOH_AVG: cups or beers consumed on average when drinking alcohol, exrcs: free physical activity, DBP: diastolic blood pressure, Waist: waist circumference, BMI: Body Mass Index, WHtR: Waist-to-Height-Ratio, SBP: systolic blood pressure. (B) cola_soda: medium cola soda,_c_coffe: a cup of coffee, tortilla: corn tortilla, flav_water: a glass of flavored sweetened water, snore: snore during sleep, DBP: diastolic blood pressure, Waist: waist circumference, BMI: Body Mass Index, WHtR: Waist-to-Height-Ratio, SBP: systolic blood pressure. 


\subsection{Analysis and Comparison}

Once the most important variables for men and women were identified, we proceed to make an analysis and comparison of these. Table $3 \mathrm{~A}$ shows the resulting variables by category in descending order for men and women, Table $3 \mathrm{~B}$ shows the resulting variables in whole dataset.

Table 3. (A) Resulting variables by category. (B) Resulting variables in whole dataset by importance.

\begin{tabular}{|c|c|c|c|}
\hline Category & Method & Gender & Resulting variables \\
\hline \multicolumn{4}{|l|}{ (A) } \\
\hline Feeding habits & VIM of RF & women & $\begin{array}{l}\text { c_coffe, cola_soda, flav_water, flav_soda, } \\
\text { and oax_cheese }\end{array}$ \\
\hline Feeding habits & VIM of RF & men & $\begin{array}{l}\text { c_coffe, cola_soda, flav_water, hot_chili, } \\
\text { and tortilla }\end{array}$ \\
\hline Quality of sleep & VIM of RF & women & $\begin{array}{l}\text { drowsy, nt_sleep, restless, snore, and } \\
\text { lit_sleep }\end{array}$ \\
\hline Quality of sleep & VIM of RF & men & drowsy, nt_sleep, restless, snore, and tired \\
\hline Anthropometry & VIM of RF & women & Age, BMI, DBP, SBP, Waist, and WHtR \\
\hline Anthropometry & VIM of RF & men & Age, BMI, DBP, SBP, Waist, and WHtR \\
\hline Habits & VIM of RF & women & EtOH_AVG, exrcs, and smk.smke \\
\hline Habits & VIM of RF & men & EtOH_AVG, exrcs, and smk.smke \\
\hline \multicolumn{4}{|l|}{ (B) } \\
\hline \multirow{6}{*}{ Whole Dataset } & VIM of RF & women & $\begin{array}{l}\text { Age, Waist, BMI, WHtR, Weight, Height, } \\
\text { SBP, DBP, cola_soda, snore, oax_cheese } \\
\text { and c_coffe }\end{array}$ \\
\hline & VIM of RF & men & $\begin{array}{l}\text { DBP, Waist, BMI, VHtR, SBP, Weight, } \\
\text { Age, snore, cola_soda, flav_water, } \\
\text { c_coffe,and tortilla }\end{array}$ \\
\hline & PCC & women & $\begin{array}{l}\text { Age, Weight, BMI, Waist, WHtR, SBP, } \\
\text { DBP, snore, cola_soda, and pork_r }\end{array}$ \\
\hline & PCC & men & $\begin{array}{l}\text { Age, Weight, BMI, Waist, WHtR, SBP, } \\
\text { DBP, snore, and cola_soda }\end{array}$ \\
\hline & Chi.square & women & $\begin{array}{l}\text { Age, BMI, Waist, WHtR, Weight, SBP, } \\
\text { DBP, snore and cola_soda }\end{array}$ \\
\hline & Chi.square & men & $\begin{array}{l}\text { BMI, Waist, DBP, SBP, Weight, WHtR, } \\
\text { Age, snore and cola_soda }\end{array}$ \\
\hline
\end{tabular}

(A) cola_soda: medium cola soda,flav_soda: soda,flav_water: a glass of flavored sweetened water, oax_cheese: Oaxaca cheese, hot_chili: a tablespoon of hot sauce or chili in food, c_coffe: a cup of coffee, tortilla: corn tortilla, flav_water: a glass of flavored sweetened water, tortilla: corn tortilla, snore: snore during sleep, restless: restless sleep, nt_sleep: not getting enough sleep, lit_sleep: little naps, drowsy: feeling drowsy, tired: feel fatigue, DBP: diastolic blood pressure, Waist: waist circumference, BMI: Body Mass Index, WHtR: Waist-to-Height-Ratio, SBP: systolic blood pressure, EtOH_AVG: cups or beers consumed on average when drinking, smk.smke: smoke and exrcs: free physical activity. (B) cola_soda: medium cola soda, flav_soda: soda,flav_water: a glass of flavored sweetened water, oax_cheese: Oaxaca cheese, hot_chili: a tablespoon of hot sauce or chili in food, c_coffe: a cup of coffee, tortilla: corn tortilla, snore: snore during sleep, restless: restless sleep, nt_sleep: not getting enough sleep, lit_sleep: little naps, drowsy: feeling drowsy, tired: feel fatigue, DBP: diastolic blood pressure. Waist: waist circumference, BMI: Body Mass Index, WHtR: Waistto-Height-Ratio, SBP: systolic blood pressure, EtOH_AVG: cups or beers consumed on average when drinking, smk.smke: smoke and excrs: free physical activity.

\subsection{Performance Evaluation of Classifiers}

This subsection describes the resulting features after the selection; it means that the variables that contribute to the classifier model; the materials and methods section details the characteristics of each one, these variables can be a dichotomous or nominal numerical value, and it is related to their parameters.

Based on the resulting variables by category and those that were obtained from the whole dataset, we developed several models in order to test their performance evaluation and identify the relevant features that support the prediction of MetS without blood screening.

In order to evaluate the resulting variables in whole dataset that was obtained by RF, PCC and Chi.square, we applied RF, deep neural network, and C4.5, as well as RF and deep neural network for resulting variables by category. 
In case of RF, the value of ntree varied between 100 to 1000 (ntree $=100,200,300$, 500,800 , and 1000); likewise, the value of the mtry (size of the random subsets of variables considered for splitting) also varied between 1 to 10; in both cases, the grid search method, as proposed by Hsu et al. [49], was used. For each case where RF and C4.5 were applied, we used 10-fold cross-validation with ten repeats to train the model and ensure the variation of the data. The deep neural network model was trained with 2500 epochs and Adam optimization.

Table 4 shows the results that were obtained by the classifiers with the resulting variables in whole dataset (see Table 1B) and those obtained by category (see Table 1A), using ACC, B.ACC, PPV, and NPV as evaluation metrics, as well as their respective standard deviations of the average performance for the 30 models generated for each case.

The obtained results showed that the highest value of ACC (84.12\% with an SD of 0.38) was achieved by RF, using the resulting variables obtained in whole dataset, which are: waist, WHtR, DBP, BMI, SBP, weight, age, height, cola_soda, a glass of whole milk (milk), snore, c_coffe, and oax_cheese.

The deep neural network had the best performance in B.ACC, with a value of $63.26 \%$ and an SD of 2.42, also using the resulting variables obtained in whole dataset.

In the case of PPV, the RF obtained the best performance with a value of $85.73 \%$ and an SD of 0.32 , using the resulting variables obtained by category, which are: waist, WHtR, BMI, SBP, DBP, age, cola_soda, flav_water, c_coffe, snore, nt_sleep, restless, flav_soda, and drowsy.

Regarding NPV, the deep neural network achieved the best performance with a value of $85.76 \%$ and an SD of 1.11, again with the whole dataset's relevant variables.

Considering the results that were obtained in the metrics by the different models, it is possible to identify that the first RF model with an mtry $=1$ and ntree $=300$ has the best performance using the resulting variables from the whole dataset (Waist, WHtR, DBP, BMI, SBP, Weight, Age, Height, cola_soda, milk, snore, c_coffe, and oax_cheese), even though the deep neural network with the same variables has a better performance in en B.ACC $(63.26 \%)$; the difference is minimal $(0.33 \%)$. Similarly, despite the fact that the RF model that uses the resulting variables by category has the highest performance in PPV $(85.73 \%)$, the difference is also minimal $(0.95 \%)$.

Concerning men, Table 5 shows the results of performance evaluation with the results variables by category and from whole dataset applying RF, deep neural network, and C4.5, using ACC, B.ACC, PPV, and NPV as evaluation metrics, as well as their respective standard deviations of the average performance for the 30 models that are generated for each case. In this case, the results obtained by the classifiers were better than those that were obtained with women data.

The RF obtained the best values in ACC (88.17\% and an SD of 0.49$)$, B.ACC $(80.73 \%$ and an SD of 0.84), and PPV (92.29\% and an SD of 0.36) using the variables that were obtained from the whole dataset, such as: Waist, DBP, SBP, BMI, WHtR, Weight, Height, Age, c_coffe, flav_water, tortilla, cola_soda, and snore. With respect to the best value of NPV, it was obtained by the deep neural network (91.26\% and an SD of 1.79) using the variables obtained by PCC in the whole dataset, which are: age, weight, BMI, waist, WHtR, SBP, DBP, snore, cola_soda, and tortilla. In this case, the RF model presents a better performance, although its NPV was not a high as other classifiers, such as the deep neural network, and it does obtain the highest value in B.ACC, thus obtaining a better performance in balanced classifications. 
Table 4. Model performance in women.

\begin{tabular}{|c|c|c|c|c|c|c|}
\hline Case & Classifier & Features & $\operatorname{ACC}(\%)$ & B.ACC (\%) & PPV (\%) & NPV (\%) \\
\hline \multirow[t]{2}{*}{$\begin{array}{l}\text { Whole dataset with } \\
\text { RF }\end{array}$} & $\begin{array}{l}\text { Random forest Mtry } \\
\quad=1 \text { Ntree }=300\end{array}$ & $\begin{array}{c}\text { Waist, WHtR, DBP, BMI, SBP, Weight, Age, } \\
\text { Height, cola_soda, milk, snore, c_coffe, } \\
\text { FREC009, oax_cheese }\end{array}$ & $84.12 \pm 0.38$ & $62.93 \pm 0.81$ & $84.78 \pm 0.28$ & $75.92 \pm 2.81$ \\
\hline & $\begin{array}{l}\text { Deep neural } \\
\text { network }\end{array}$ & $\begin{array}{l}\text { Waist, WHtR, DBP, BMI, SBP, Weight, Age, } \\
\text { Height, cola_soda, milk, snore, c_coffe, } \\
\text { FREC009, oax_cheese }\end{array}$ & $80.03 \pm 1.04$ & $63.26 \pm 2.42$ & $50.78 \pm 3.38$ & $85.76 \pm 1.11$ \\
\hline \multirow[t]{2}{*}{$\begin{array}{l}\text { Whole dataset with } \\
\text { PCC }\end{array}$} & $\begin{array}{l}\text { Deep neural } \\
\text { network }\end{array}$ & $\begin{array}{l}\text { Age, Weight, BMI, Waist, WhtR, SBP, DBP, snore, } \\
\text { cola_soda, FREQ032 }\end{array}$ & $81.06 \pm 0.75$ & $62.4 \pm 3.22$ & $56.88 \pm 5.61$ & $84.43 \pm 1.38$ \\
\hline & $\begin{array}{l}\text { Random forest Mtry } \\
\quad=1 \text { Ntree }=500\end{array}$ & $\begin{array}{l}\text { Age, Weight, BMI, Waist, WhtR, SBP, DBP, snore, } \\
\text { cola_soda, FREQ032 }\end{array}$ & $83.88 \pm 0.30$ & $62.63 \pm 0.54$ & $84.99 \pm 0.19$ & $70.62 \pm 2.22$ \\
\hline $\begin{array}{l}\text { Whole dataset with } \\
\text { chi.square }\end{array}$ & C4.5 & $\begin{array}{l}\text { Waist, WhtR, BMI, DBP, SBP, Age, snore, } \\
\text { cola_soda }\end{array}$ & $83.35 \pm 0.11$ & $61.91 \pm 0.33$ & $84.82 \pm 0.13$ & $72.19 \pm 1.24$ \\
\hline \multirow[t]{2}{*}{$\begin{array}{l}\text { Resulting variables } \\
\text { by category }\end{array}$} & $\begin{array}{l}\text { Random forest Mtry } \\
\quad=3 \text { Ntree }=100\end{array}$ & $\begin{array}{l}\text { Waist, WHtR, BMI, SBP, DBP, Age, cola_soda, } \\
\text { flav_water, c_coffe, snore, nt_sleep, restless, } \\
\text { flav_soda, drowsy }\end{array}$ & $84.04 \pm 0.55$ & $64.58 \pm 0.92$ & $85.73 \pm 0.32$ & $67.80 \pm 3.33$ \\
\hline & $\begin{array}{l}\text { Deep neural } \\
\text { network }\end{array}$ & $\begin{array}{c}\text { Waist, WhtR, BMI, SBP, DBP, Age, cola_soda, } \\
\text { flav_water, c_coffe, snore, nt_sleep, restless, } \\
\text { flav_soda, drowsy, oax_cheese }\end{array}$ & $78.32 \pm 1.45$ & $63.12 \pm 1.97$ & $45.69 \pm 4.08$ & $84.96 \pm 0.85$ \\
\hline
\end{tabular}

cola_soda: medium cola soda, milk: a glass of whole milk, c_coffe: a cup of coffee, FREC009: an orange, oax_cheese: Oaxaca cheese,flav_soda: flavored soda, flav_water: a glass of flavored sweetened water, pork_r : pork rind. snore: snore during sleep, $n t \_s l e e p$ : not getting enough sleep, drowsy: feeling drowsy, restless: restless sleep, tired: feel fatigue. Waist: waist circumference, BMI: Body Mass Index, WHtR: Waist-to-Height-Ratio, SBP: systolic blood pressure, DBP: diastolic blood pressure.

Table 5. Model performance in men.

\begin{tabular}{|c|c|c|c|c|c|c|}
\hline Case & Classifier & Features & $\operatorname{ACC}(\%)$ & B.ACC (\%) & PPV (\%) & NPV (\%) \\
\hline \multirow[t]{2}{*}{$\begin{array}{l}\text { Whole datase with } \\
\text { RF }\end{array}$} & $\begin{array}{l}\text { Random forest Mtry } \\
\quad=10 \text { Ntree }=800\end{array}$ & $\begin{array}{l}\text { Waist, DBP, SBP, BMI, WHtR, Weight, Height, } \\
\text { Age, cola_soda, c_coffe, flav_water, tortilla, } \\
\text { snore }\end{array}$ & $88.17 \pm 0.49$ & $80.73 \pm 0.84$ & $92.29 \pm 0.36$ & $70.72 \pm 2.81$ \\
\hline & $\begin{array}{l}\text { Deep neural } \\
\text { network }\end{array}$ & $\begin{array}{l}\text { Waist, DBP, SBP, BMI, WHtR, Weight, Height, } \\
\text { Age, cola_soda, c_coffe, flav_water, tortilla, } \\
\text { snore }\end{array}$ & $85.30 \pm 0.88$ & $73.01 \pm 4.86$ & $59.06 \pm 6.00$ & $90.77 \pm 2.09$ \\
\hline \multirow[t]{2}{*}{$\begin{array}{l}\text { Complete dataset } \\
\text { with PCC }\end{array}$} & $\begin{array}{l}\text { Deep neural } \\
\text { network }\end{array}$ & $\begin{array}{c}\text { Age, Weight, BMI, Waist, WHtR, SBP, DBP, } \\
\text { snore, cola_soda }\end{array}$ & $86.38 \pm 0.60$ & $74.63 \pm 4.28$ & $61.75 \pm 3.54$ & $91.26 \pm 1.79$ \\
\hline & $\begin{array}{l}\text { Random forest Mtry } \\
\quad=2 \text { Ntree }=800\end{array}$ & $\begin{array}{l}\text { Age, Weight, BMI, Waist, WHtR, SBP, DBP, } \\
\text { snore, cola_soda }\end{array}$ & $83.73 \pm 0.56$ & $65.29 \pm 0.95$ & $86.05 \pm 0.34$ & $64.2 \pm 2.95$ \\
\hline $\begin{array}{l}\text { Complete dataset } \\
\text { with chi.square }\end{array}$ & C4.5 & $\begin{array}{l}\text { Waist, WHtR, BMI, DBP, SBP, Age, snore, } \\
\text { cola_soda }\end{array}$ & $86.38 \pm 0.12$ & $74.12 \pm 0.38$ & $89.42 \pm 0.18$ & $71.61 \pm 0.78$ \\
\hline \multirow[t]{2}{*}{$\begin{array}{l}\text { Resulting variables } \\
\text { by category }\end{array}$} & $\begin{array}{l}\text { Random forest Mtry } \\
\quad=9 \text { Ntree }=800\end{array}$ & $\begin{array}{c}\text { Waist, DBP, BMI, SBP, WHtR, Age, c_coffe, } \\
\text { flav_water, hot_chili, tortilla, drowsy, cola_soda, } \\
\text { restless, snore, nt_sleep }\end{array}$ & $87.47 \pm 0.27$ & $78.94 \pm 0.40$ & $91.49 \pm 0.18$ & $69.65 \pm 1.04$ \\
\hline & $\begin{array}{l}\text { Deep neural } \\
\text { network }\end{array}$ & $\begin{array}{c}\text { Waist, DBP, BMI, SBP, WHtR, Age, c_coffe, } \\
\text { flav_water, hot_chili, tortilla, drowsy, cola_soda, } \\
\text { restless, snore, nt_sleep }\end{array}$ & $84.96 \pm 1.34$ & $72.52 \pm 2.89$ & $57.75 \pm 5.58$ & $90.13 \pm 1.19$ \\
\hline
\end{tabular}

cola_soda: medium cola soda, c_coffe: a cup of coffee, flav_water: a glass of flavored sweetened water, tortilla: corn tortilla, hot_chili: a tablespoon of hot sauce or chili in food. snore: snore during sleep, nt_sleep: not getting enough sleep, drowsy: feeling drowsy, restless: restless sleep, tired: feel fatigue. Waist: waist circumference, DBP: diastolic blood pressure. SBP: systolic blood pressure, BMI: Body Mass Index, WHtR: Waist-to-Height-Ratio.

The relevant variables that were found by the Chi.Square filter method, both for women (Table 1B) and for men (Table 2B), were used to create predictive models with the C4.5 classifier to compare them with the models that were created with Deep Neural Network and Random Forest. Furthermore, C4.5 has the advantage of creating a model that is interpretable to the naked eye by a person. In Figure 3, we present the best model for women and in Figure 4, the best model for men, both being found over 30 independent runs using the train set. Classification trees have the property of making an embedded variable selection during the predictive model creation process. In the case of women, the variables that were selected for the construction of the tree were WAIST, SBP, DBP, AGE, $\mathrm{BMI}$, and FREC080. For men, the variables selected for the construction of the tree were WAIST, SBP, DBP, WhtR, and BMI.

The model shown in Figure 3 represents the best predictive model created with C4.5 for women across 30 independent runs. For this model, using data from the train set, it was found that, when the WAIST variable is greater than or equal to 89 and the DBP variable is greater than or equal to $85,92 \%$ of the patients suffer from MS, when considering $4 \%$ of the cases in the training set. 


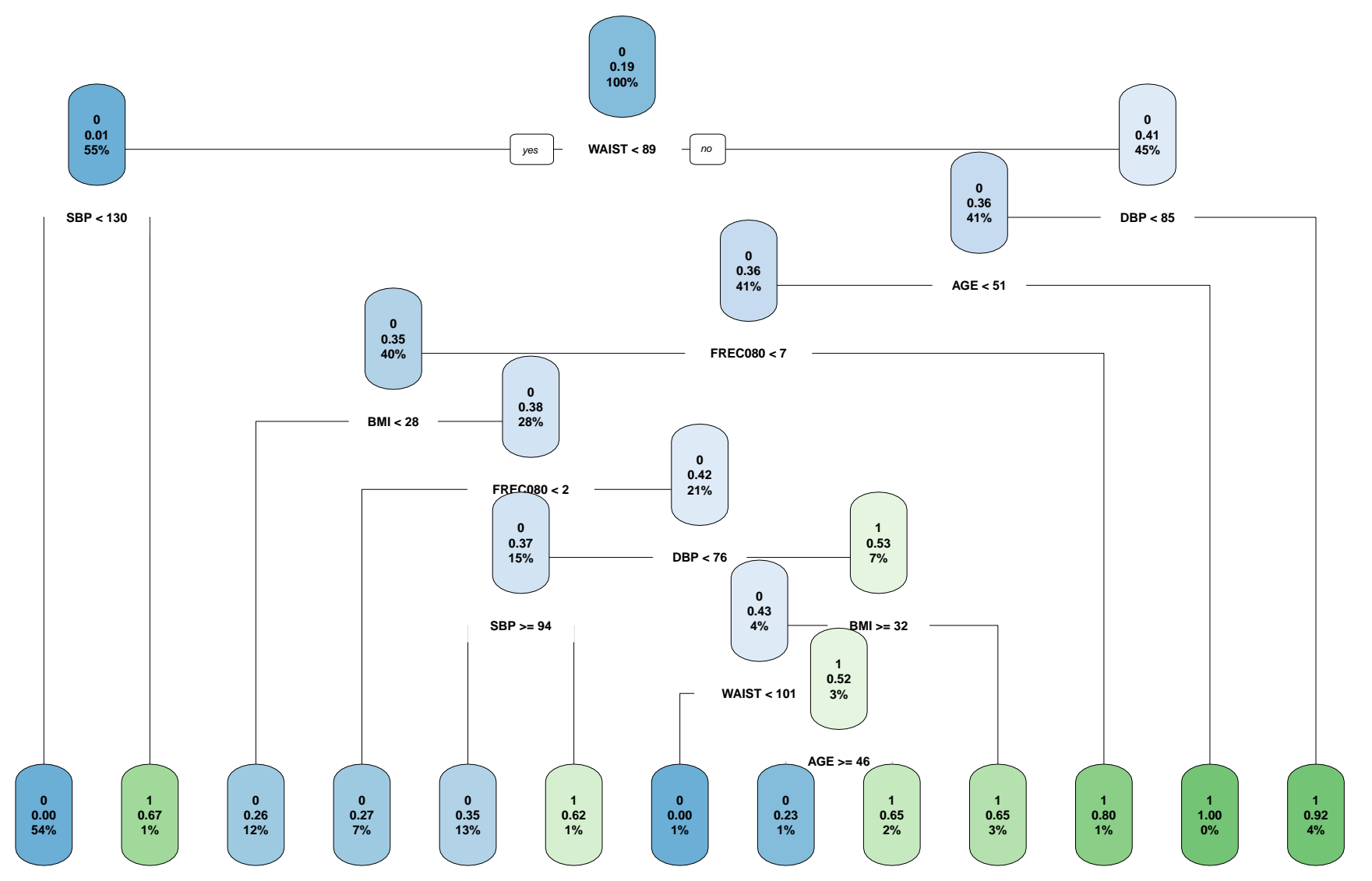

Figure 3. Best predictive model built with C4.5 for women across 30 independent runs.

The model presented in Figure 4 represents the best predictive model created with C4.5 for men across 30 independent runs. For this model, using data from the train set, it was found that, when the WAIST variable is greater than or equal to 103 and the DBP variable is greater than or equal to $82,89 \%$ of the patients suffer from MS, while considering $8 \%$ of the cases in the training set.

In general, by going through the branches of the classification trees, it is possible to obtain the conditions that determine whether or not a patient will be diagnosed with MS, for both women and men.

\subsection{The Best Model}

According the results in metrics that were obtained by the classifiers, it was possible to identify the best model as well as the most relevant features for women and men.

For women, the best model was RF with a mtry of 1 and Ntree of 300, and the following features: waist, WHtR, DBP, BMI, SBP, Weight, Age, Height, cola_soda, milk, snore, c_coffe, and oax_cheese.

In men, again, the best model was RF with a mtry of 10 and Ntree of 800, which obtained the best performance using the variables: waist, DBP, SBP, BMI, WHtR, weight, height, age, cola_soda, c_coffe, flav_water, tortilla, and snore.

The relevant features obtained are strongly related with the diagnosis and the risk of developing MetS, such as consumption habits of alcoholic beverages [50], the consumption of cola soft drinks [51,52], sleep disorders, as the action of snoring when the person is sleeping [53], weight, age [54], and the recognized by ATP III, IDF, and WHO (waist, SBP, and DBP). 


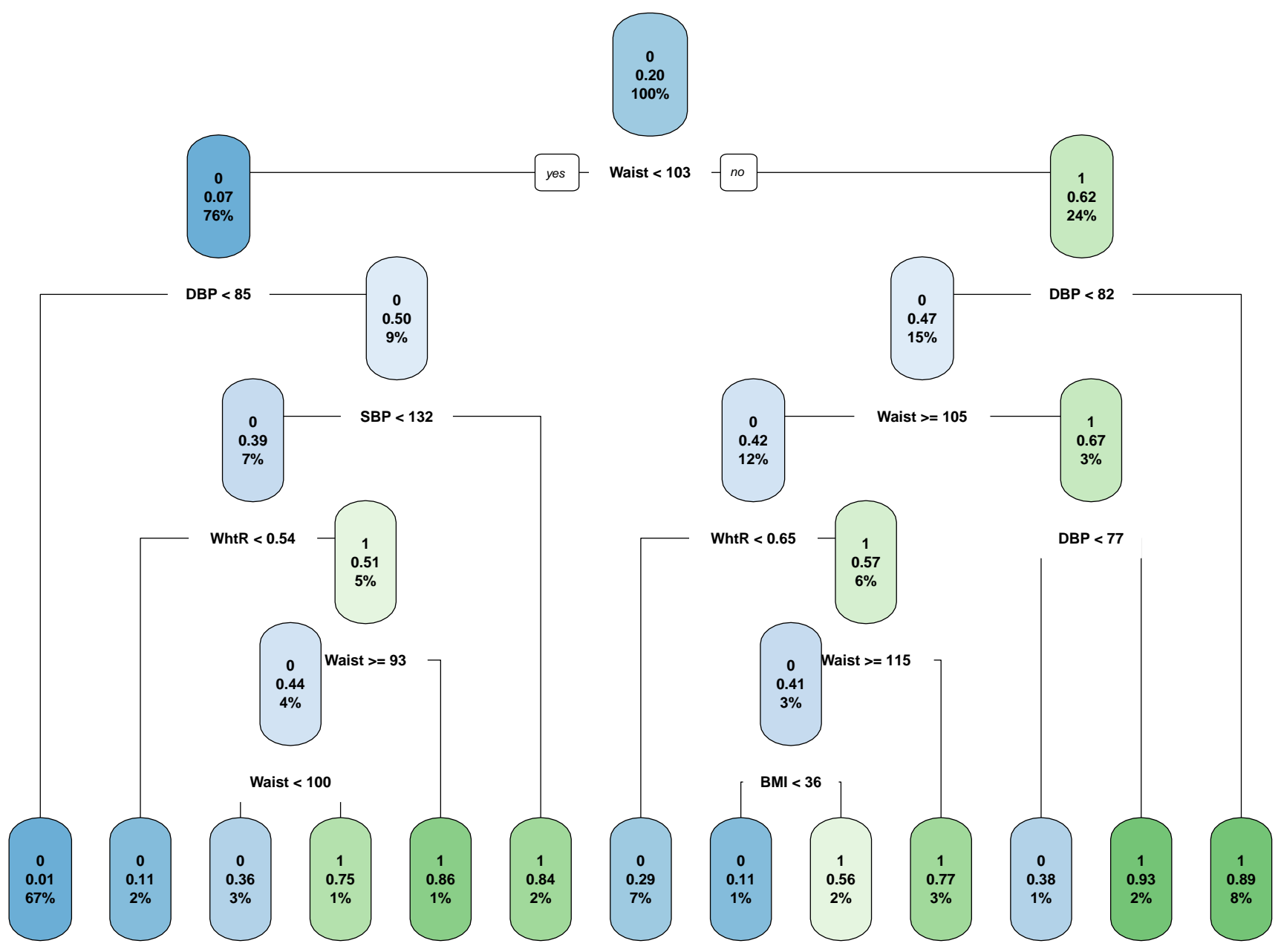

Figure 4. Best predictive model built with C4.5 for men across 30 independent runs.

\section{Discussion}

\subsection{Best Model for the Risk Calculator}

The MetS is considered to be a potential risk factor for cardiovascular disease and diabetes, it has grown exponentially in Mexico and other countries. For this reason, the development of risk calculator tools is important, especially within the prevention perspective. Based on the above, researches have developed models applying machine learning algorithms to support the diagnosis or prediction of MetS, while considering diverse definitions, such as NCEP ATP III, WHO, and IDF (three of the most used criteria throughout the world [55]), which consider health parameters that must be determined with blood screening. However, other studies $[19,21,56]$ have proved that MetS can also be diagnosed without blood studies, while taking other risk factors into consideration.

In this work, we used RF, C4.5, and DNN with the purpose of identifying risk factors that are related to anthropometric factors, sleepy manner associated with snoring, and dietary habits to predict MetS. Accordingly, [18] comparing RF with types of ANN is a prominent topic.

Each algorithm was performed by 30 independent runs, the average of ACC, B.ACC, PPV, and NPV are presented in Table 4 for women and Table 5 for men. The results for both genders shows that highest value in the metrics was obtained by the RF with a mtry $=1$ and ntree $=300$ for women $(\mathrm{ACC}=84.12 \%$, B.ACC $=62.93 \%, \mathrm{PPV}=84.78 \%$, and NPV $=75.92 \%$ ) and a $\operatorname{mtry}=10$ and ntree $=800$ for $\operatorname{men}(\mathrm{ACC}=88.17 \%$, B.ACC $=80.73 \%, \mathrm{PPV}=92.29 \%$, and NPV $=70.72 \%$ ).

Based on the results that are shown in Tables 4 and 5, it is possible to observe that RF and the DNN show comparable performances in ACC and B.ACC, but lower performance 
in PPV and high performance in NPV, however the results that are obtained by RF are close to each other, presenting a suitable solution for the prediction of MetS. The optimal model of RF was performed analyzing several models with ntree between 100 to 1000 trees and mtry between 1 to 10 .

Additionally, it is essential to highlight that deep learning models do not require an earlier feature selection step; they get the features from the data; even the above is a property for DL, a better performance was obtained by selecting features by PCC, RF by category, and all characteristics. One test was carried on with all of the features, but this experiment had a more unsatisfactory performance; the main reason is the quantity of data; DL is more effective when the set has a large amount of information.

\subsection{Most Relevant Features}

According to the literature [54,57], weight, waist, age, diastolic, and systolic blood pressure are risk factors that are considered for the diagnosis of MetS. Likewise, in this work, we found other risk factors for men and women that have also been studied due to their relationship with MetS and obesity.

In the case of women, the best model (RF with a mtry $=1$ and ntree $=300$ ) showed that variables related to obesity, such as waist circumference and WHtR, were the ones that obtained the highest value in terms of importance; followed by the DBP and SBP, the age and the height, trouble sleeping associated with snoring, restless sleep, and somnolence. Likewise, regarding the dietary habits, we identify that women with MetS have a high consumption of cola soda, coffee, whole milk, oranges, flavored sweetened water, and flavor soda.

For men, the best model (RF with a mtry $=10$ and ntree $=800)$ showed that the waist circumference and blood pressure (DBP and SBP) were the highest risk factors, followed by the BMI, the WHtR, the weight, and the age. Regarding the dietary habits, it was possible to identify that men with MetS preferably consume cola soda, coffee, flavored sweetened water, and corn tortilla. Additionally, like women, men have sleep problems, with snoring being the risk factor outstanding.

As can be seen in the results section, in the case of men, more specific features were revealed; therefore, variable importance is reflected in those that are related to the blood pressure (SBP and DBP). Moreover, classifiers' performance was high; RF was the best in ACC average of $88.17 \%$, a B.ACC of $80.73 \%$, a PPV of $92.29 \%$, and an NPV of $70.72 \%$. In the case of women, the variable importance denotes a close relationship with obesity, with RF being the best classifier with an ACC of $84.12 \%$, a B.ACC of $62.93 \%$, a PPV of $84.78 \%$, and an NPV $75.92 \%$.

The most relevant features identified in this work as prognostic variables to predict MetS in Mexican women and men were strongly related to this syndrome. When the person is sleeping, the case of snoring has been a potential factor that is strongly related to obesity and the risk of suffering MetS [58]. Recent work also suggests a strong association with MetS, even when the snore eliminates the repeated apnea and hypoxia; simple snoring was still strongly associated with MetS [59]. According to studies [60,61], Mexico is one of the countries with a high consumption of sugary drinks. This study found that cola soda, flavored sweetened water, and flavor soda were common consumption habits in both men and women. However, these drinks contribute positively to the risk of developing obesity and chronic diseases [62]. Likewise, tortilla consumption has been associated with the prevalence of overweight, obesity, and MetS in Mexican adults. Coffee was another consumed beverage by both genders; nevertheless, recent studies $[63,64]$ have reported that coffee consumption was not significantly associated with metabolic syndrome [65].

Based on these results, this work could be use for the prevention of MetS. The population can access a simple survey of the healthcare system monitoring. If some risk factors are detected, the people can be directed to medical revision; the above might prevents future problems, by reducing the cost in laboratory tests and treatments. 


\subsection{Limitations}

This research was based on data that were obtained from a cohort of relatively healthy adult residents of Mexico City.

\section{Conclusions}

In this study, different machine learning algorithms were applied; nevertheless, RF obtained the highest performance to identify the best features by gender and predict MetS without an invasive study or laboratory tests. It should be noted that RF has been one of the best machine learning algorithms to predict the MetS $[18,19]$.

The prognostic variables that were found in both genders were positively related to obesity, blood pressure, and MetS; besides, they can be obtained in a first medical consultation and can be monitored thoroughly. Besides, the separation by gender allowed for discovering differences in dietary patterns, which can be associated with risk factors associated with the development MetS.

Although, in this study, we found a group of risk factors that support the prediction of MetS, we consider it essential to expand the data-set with data from other regions of Mexico, where diet could vary, as well as lifestyles.

Furthermore, this work implements machine learning models, and it lays the foundation to program a friendly graphic interface, including a calculator, in order to bring health monitoring tools. Additionally, implementation is recommended to be carried out directly with the obtained model since developing the equation among the obtained weights and the interaction between trees or layers.

Author Contributions: Conceptualization-G.O.G.-E., M.V.; Methodology-G.O.G.-E., T.A.R.-d., J.H.-T.; Software-G.O.G.-E., T.A.R.-d., J.H.-T.; Supervision-M.V.; Validation-G.O.G.-E., T.A.R.-d.; formal analysis-J.H.-T,; writing—original draft preparation-G.O.G.-E., M.M.-G., J.H.-T., M.V.; writing-review and editing-G.O.G.-E., T.A.R.-d., J.H.-T., M.M.-G., M.V., O.I.V. All authors have read and agreed to the published version of the manuscript.

Funding: This research was supported by the National Council of Science and Technology (CONACYT, México), Cátedras CONACYT 1591.

Institutional Review Board Statement: The study was conducted according to the guidelines of the Declaration of Helsinki, and approved by the Institutional Review Board (or Ethics Committee) of Instituto Nacional de Cardiología-Ignacio Chavez (INC-ICh)(protocol code 13-802 and approval on 23 September 2014).

Informed Consent Statement: Informed consent was obtained from all subjects involved in the study.

Data Availability Statement: All data presented used in this study are openly available from: https://github.com/taniaglae/mets (accessed on 28 April 2021).

Acknowledgments: We want to extend our appreciation to Consejo Nacional de Ciencia y Tecnología (CONACYT) (National Council for Science and Technology) under the 'Cátedras CONACYT' programme, No. 1591.

Conflicts of Interest: The authors declare no conflict of interest.

\section{References}

1. Eckel, R.H.; Grundy, S.M.; Zimmet, P.Z. The metabolic syndrome. Lancet 2005, 365, 1415-1428. [CrossRef]

2. Gutiérrez-Solis, A.L.; Datta Banik, S.; Méndez-González, R.M. Prevalence of metabolic syndrome in mexico: A systematic review and meta-analysis. Metab. Syndr. Relat. Disord. 2018, 16, 395-405. [CrossRef] [PubMed]

3. Moore, J.X.; Chaudhary, N.; Akinyemiju, T. Peer reviewed: Metabolic syndrome prevalence by race/ethnicity and sex in the United States, National Health and Nutrition Examination Survey, 1988-2012. Prev. Chronic Dis. 2017, 14, E24. [CrossRef] [PubMed]

4. Grundy, S.M.; Brewer, H.B., Jr.; Cleeman, J.I.; Smith, S.C., Jr.; Lenfant, C. Definition of metabolic syndrome: Report of the National Heart, Lung, and Blood Institute/American Heart Association conference on scientific issues related to definition. Circulation 2004, 109, 433-438. [CrossRef] [PubMed] 
5. Alberti, K.G.M.; Zimmet, P.; Shaw, J. The metabolic syndrome-A new worldwide definition. Lancet 2005, 366, 1059-1062. [CrossRef]

6. Alberti, K.G.M.M.; Zimmet, P.; Shaw, J. Metabolic syndrome-A new world-wide definition. A consensus statement from the international diabetes federation. Diabet. Med. 2006, 23, 469-480. [CrossRef] [PubMed]

7. World Health Organization (WHO). Definition, Diagnosis and Classification of Diabetes Mellitus and Its Complications: Report of a WHO Consultation. Part 1, Diagnosis and Classification of Diabetes Mellitus; Technical Report; World Health Organization: Geneva, Switzerland, 1999.

8. Choe, E.K.; Rhee, H.; Lee, S.; Shin, E.; Oh, S.W.; Lee, J.E.; Choi, S.H. Metabolic Syndrome Prediction Using Machine Learning Models with Genetic and Clinical Information from a Nonobese Healthy Population. Genom. Inform. 2018, 16, e31. [CrossRef]

9. Salazar, N.A.S.; Oviedo, L.M.V.; Samamé, L.T.; Tell, N.M. Conocimientos sobre síndrome metabólico en pacientes con sobrepeso u obesidad de un hospital de alta complejidad de lambayeque, 2016. Rev. Exp. Med. Hosp. Reg. Lambayeque REM 2018, 4, 56-60.

10. Oh, E.G.; Bang, S.Y.; Hyun, S.S.; Chu, S.H.; Jeon, J.Y.; Kang, M.S. Knowledge, perception and health behavior about metabolic syndrome for an at risk group in a rural community area. J. Korean Acad. Nurs. 2007, 37, 790-800. [CrossRef]

11. Yahia, N.; Brown, C.; Rapley, M.; Chung, M. Assessment of college students' awareness and knowledge about conditions relevant to metabolic syndrome. Diabetol. Metab. Syndr. 2014, 6, 111. [CrossRef]

12. Liu, X.; Faes, L.; Kale, A.U.; Wagner, S.K.; Fu, D.J.; Bruynseels, A.; Mahendiran, T.; Moraes, G.; Shamdas, M.; Kern, C.; et al. A comparison of deep learning performance against health-care professionals in detecting diseases from medical imaging: A systematic review and meta-analysis. Lancet Digit. Health 2019, 1, e271-e297. [CrossRef]

13. Maity, N.G.; Das, S. Machine learning for improved diagnosis and prognosis in healthcare. In Proceedings of the 2017 IEEE Aerospace Conference, Big Sky, MT, USA, 4-1 March 2017; pp. 1-9.

14. Sethi, P.; Jain, M. A comparative feature selection approach for the prediction of healthcare coverage. In International Conference on Information Systems, Technology and Management; Springer: Berlin/Heidelberg, Germany, 2010; pp. 392-403.

15. Jain, D.; Singh, V. Feature selection and classification systems for chronic disease prediction: A review. Egypt. Inform. J. 2018, 19, 179-189. [CrossRef]

16. Foster, K.R.; Koprowski, R.; Skufca, J.D. Machine learning, medical diagnosis, and biomedical engineering research-commentary. Biomed. Eng. Online 2014, 13, 94. [CrossRef] [PubMed]

17. Worachartcheewan, A.; Shoombuatong, W.; Pidetcha, P.; Nopnithipat, W.; Prachayasittikul, V.; Nantasenamat, C. Predicting metabolic syndrome using the random forest method. Sci. World J. 2015, 2015. [CrossRef]

18. Vrbaški, D.; Vrbaški, M.; Kupusinac, A.; Ivanović, D.; Stokić, E.; Ivetić, D.; Doroslovački, K. Methods for algorithmic diagnosis of metabolic syndrome. Artif. Intell. Med. 2019, 101, 101708. [CrossRef]

19. Barrios, M.; Jimeno, M.; Villalba, P.; Navarro, E. Novel Data Mining Methodology for Healthcare Applied to a New Model to Diagnose Metabolic Syndrome without a Blood Test. Diagnostics 2019, 9, 192. [CrossRef]

20. Murguía-Romero, M.; Jiménez-Flores, R.; Méndez-Cruz, A.R.; Villalobos-Molina, R. Predicting metabolic syndrome with neural networks. In Mexican International Conference on Artificial Intelligence; Springer: Berlin/Heidelberg, Germany, 2013 ; pp. 464-472.

21. Ivanović, D.; Kupusinac, A.; Stokić, E.; Doroslovački, R.; Ivetić, D. ANN prediction of metabolic syndrome: A complex puzzle that will be completed. J. Med. Syst. 2016, 40, 264. [CrossRef]

22. Wirfält, E.; Hedblad, B.; Gullberg, B.; Mattisson, I.; Andrén, C.; Rosander, U.; Janzon, L.; Berglund, G. Food patterns and components of the metabolic syndrome in men and women: a cross-sectional study within the Malmö Diet and Cancer cohort. Am. J. Epidemiol. 2001, 154, 1150-1159. [CrossRef]

23. Panagiotakos, D.B.; Pitsavos, C.; Skoumas, Y.; Stefanadis, C. The association between food patterns and the metabolic syndrome using principal components analysis: The ATTICA Study. J. Am. Diet. Assoc. 2007, 107, 979-987. [CrossRef]

24. Sarebanhassanabadi, M.; Mirhosseini, S.J.; Mirzaei, M.; Namayandeh, S.M.; Soltani, M.H.; Pakseresht, M.; Pedarzadeh, A.; Baramesipour, Z.; Faraji, R.; Salehi-Abargouei, A. Effect of dietary habits on the risk of metabolic syndrome: Yazd Healthy Heart Project. Public Health Nutr. 2018, 21, 1139-1146. [CrossRef]

25. Elmadhun, N.Y.; Sellke, F.W. Is there a link between alcohol consumption and metabolic syndrome? Clin. Lipidol. 2013, 8, 5-8. [CrossRef]

26. Jia, W.P. The impact of cigarette smoking on metabolic syndrome. Biomed. Environ. Sci. 2013, 26, 947-952.

27. Nambiar, L.; Bhimjiyani, A.; Khandelwal, S. A systematic review to assess the impact of physical activity intervention on people with metabolic syndrome. J. Sci. Med. Sport 2014, 18, e117. [CrossRef]

28. Fernandez-Mendoza, J.; He, F.; LaGrotte, C.; Vgontzas, A.N.; Liao, D.; Bixler, E.O. Impact of the metabolic syndrome on mortality is modified by objective short sleep duration. J. Am. Heart Assoc. 2017, 6, e005479. [CrossRef]

29. Colín-Ramírez, E.; Rivera-Mancía, S.; Infante-Vázquez, O.; Cartas-Rosado, R.; Vargas-Barrón, J.; Madero, M.; Vallejo, M. Protocol for a prospective longitudinal study of risk factors for hypertension incidence in a Mexico City population: The Tlalpan 2020 cohort. BMJ Open 2017, 7, e016773. [CrossRef]

30. Chobanian, A.V.; Bakris, G.L.; Black, H.R.; Cushman, W.C.; Green, L.A.; Izzo, J.L., Jr.; Jones, D.W.; Materson, B.J.; Oparil, S.; Wright, J.T., Jr.; et al. Seventh report of the joint national committee on prevention, detection, evaluation, and treatment of high blood pressure. Hypertension 2003, 42, 1206-1252. [CrossRef]

31. Marfell-Jones, M.J.; Stewart, A.; De Ridder, J. International Standards for Anthropometric Assessment; International Society for the Advancement of Kinanthropometry: Wellington, New Zealand, 2012. 
32. Hernández-Avila, J. gonzález-Avilés, L, Rosales-Mendoza, E. Manual de Usuario. SNUT Sistema de Evaluación de Hábitos Nutricionales y Consumo de Nutrimentos; Instituto Nacional de Salud Pública: Cuernavaca, Mexico, 2003.

33. Craig, C.L.; Marshall, A.L.; Sjöström, M.; Bauman, A.E.; Booth, M.L.; Ainsworth, B.E.; Pratt, M.; Ekelund, U.; Yngve, A.; Sallis, J.F.; others. International physical activity questionnaire: 12-country reliability and validity. Med. Sci. Sport. Exerc. 2003, 35, $1381-1395$. [CrossRef]

34. Stewart, A.L.; Ware, J.E. Measuring Functioning and Well-Being: The Medical Outcomes Study Approach; Duke University Press: Durham, NC, USA, 1992.

35. Spritzer, K.; Hays, R. MOS Sleep Scale: A Manual for Use and Scoring, Version 1.0; RAND: Los Angeles, CA, USA, 2003 ; pp. 1-8.

36. Chen, Z.; He, N.; Huang, Y.; Qin, W.T.; Liu, X.; Li, L. Integration of a deep learning classifier with a random forest approach for predicting malonylation sites. Genom. Proteom. Bioinform. 2018, 16, 451-459. [CrossRef]

37. R Core Team. R: A Language and Environment for Statistical Computing; R Foundation for Statistical Computing: Vienna, Austria, 2013.

38. Wayne, D. Bioestadística: Base Para el Análisis de las Ciencias de la Salud; Technical Report; Limusa: Ciudad de Mexico, Mexico, 1983.

39. GECCO. Genetic and Evolutionary Computation-GECCO 2003. In Proceedings of the Genetic and Evolutionary Computation Conference, Chicago, IL, USA, 12-16 July 2003; Springer: Berlin/Heidelberg, Germany, 2003.

40. Breiman, L. Random forests. Mach. Learn. 2001, 45, 5-32. [CrossRef]

41. Strobl, C.; Boulesteix, A.L.; Kneib, T.; Augustin, T.; Zeileis, A. Conditional variable importance for random forests. BMC Bioinform. 2008, 9, 307. [CrossRef]

42. Quinlan, J.R. C4.5: Programs for Machine Learning; Morgan Kaufmann Publishers Inc.: San Francisco, CA, USA, 1993.

43. Karegowda, A.; Manjunath, A.; Jayaram, M.A. Comparative study of attribute selection using gain ratio and correlation based feature selection. Int. J. Inf. Technol. Knowl. Manag. 2010, 2, 271-277.

44. Zheng, Z.; Wu, X.; Srihari, R.K. Feature selection for text categorization on imbalanced data. SIGKDD Explor. 2004, 6, 80-89. [CrossRef]

45. Benesty, J.; Chen, J.; Huang, Y.; Cohen, I. Pearson correlation coefficient. In Noise Reduction in Speech Processing; Springer: Berlin/Heidelberg, Germany, 2009; pp. 1-4.

46. Bobadilla, J.; Ortega, F.; Hernando, A. A collaborative filtering similarity measure based on singularities. Inf. Process. Manag. 2012, 48, 204-217. [CrossRef]

47. Hinton, G.E.; Osindero, S.; Teh, Y.W. A fast learning algorithm for deep belief nets. Neural Comput. 2006, 18, 1527-1554. [CrossRef]

48. Chollet, F. Keras. 2015. Available online: https:// keras.io (accessed on 20 September 2020).

49. Hsu, C.W.; Chang, C.C.; Lin, C.J. A Practical Guide to Support Vector Classification; National Taiwan University: Taipei, Taiwan, 2003.

50. Sun, K.; Ren, M.; Liu, D.; Wang, C.; Yang, C.; Yan, L. Alcohol consumption and risk of metabolic syndrome: A meta-analysis of prospective studies. Clin. Nutr. 2014, 33, 596-602. [CrossRef]

51. Høstmark, A.T. The Oslo health study: Soft drink intake is associated with the metabolic syndrome. Appl. Physiol. Nutr. Metab. 2010, 35, 635-642. [CrossRef]

52. Milei, J.; Losada, M.O.; Llambí, H.G.; Grana, D.R.; Suárez, D.; Azzato, F.; Ambrosio, G. Chronic cola drinking induces metabolic and cardiac alterations in rats. World J. Cardiol. 2011, 3, 111. [CrossRef]

53. Troxel, W.M.; Buysse, D.J.; Matthews, K.A.; Kip, K.E.; Strollo, P.J.; Hall, M.; Drumheller, O.; Reis, S.E. Sleep symptoms predict the development of the metabolic syndrome. Sleep 2010, 33, 1633-1640. [CrossRef]

54. Alley, D.E.; Chang, V.W. Metabolic syndrome and weight gain in adulthood. J. Gerontol. Ser. Biomed. Sci. Med Sci. 2010, 65, 111-117. [CrossRef]

55. Tsai, S.S.; Chu, Y.Y.; Chen, S.T.; Chu, P.H. A comparison of different definitions of metabolic syndrome for the risks of atherosclerosis and diabetes. Diabetol. Metab. Syndr. 2018, 10, 56. [CrossRef]

56. Porchia, L.M.; Lara-Solis, B.; Torres-Rasgado, E.; Gonzalez-Mejia, M.; Ruiz-Vivanco, G.; Pérez-Fuentes, R. Validation of a non-laboratorial questionnaire to identify Metabolic Syndrome among a population in central Mexico. Rev. Panam. Salud Pública 2019, 43, e9. [CrossRef] [PubMed]

57. Ho, J.S.; Cannaday, J.J.; Barlow, C.E.; Mitchell, T.L.; Cooper, K.H.; FitzGerald, S.J. Relation of the number of metabolic syndrome risk factors with all-cause and cardiovascular mortality. Am. J. Cardiol. 2008, 102, 689-692. [CrossRef] [PubMed]

58. Wu, W.T.; Tsai, S.S.; Shih, T.S.; Lin, M.H.; Chou, T.C.; Ting, H.; Wu, T.N.; Liou, S.H. The association between obstructive sleep apnea and metabolic markers and lipid profiles. PLoS ONE 2015, 10, e0130279. [CrossRef] [PubMed]

59. Zou, J.; Song, F.; Xu, H.; Fu, Y.; Xia, Y.; Qian, Y.; Zou, J.; Liu, S.; Fang, F.; Meng, L.; et al. The relationship between simple snoring and metabolic syndrome: A cross-sectional study. J. Diabetes Res. 2019, 2019, 9578391. [CrossRef]

60. Basu, S.; McKee, M.; Galea, G.; Stuckler, D. Relationship of soft drink consumption to global overweight, obesity, and diabetes: A cross-national analysis of 75 countries. Am. J. Public Health 2013, 103, 2071-2077. [CrossRef]

61. Gertner, D.; Rifkin, L. Coca-Cola and the Fight against the Global Obesity Epidemic. Thunderbird Int. Bus. Rev. 2018, 60, 161-173. [CrossRef]

62. Hu, F.B.; Malik, V.S. Sugar-sweetened beverages and risk of obesity and type 2 diabetes: Epidemiologic evidence. Physiol. Behav. 2010, 100, 47-54. [CrossRef]

63. Baspinar, B.; Eskici, G.; Ozcelik, A. How coffee affects metabolic syndrome and its components. Food Funct. 2017, 8, $2089-2101$. [CrossRef] 
64. Nordestgaard, A.T.; Thomsen, M.; Nordestgaard, B.G. Coffee intake and risk of obesity, metabolic syndrome and type 2 diabetes: A Mendelian randomization study. Int. J. Epidemiol. 2015, 44, 551-565. [CrossRef]

65. Salas, R.; del Mar Bibiloni, M.; Ramos, E.; Villarreal, J.Z.; Pons, A.; Tur, J.A.; Sureda, A. Metabolic syndrome prevalence among Northern Mexican adult population. PLoS ONE 2014, 9, e105581. [CrossRef] 